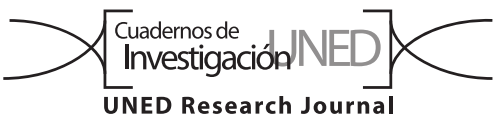

\title{
Uso de residuos agroindustriales en previveros de palma aceitera (Elaeis guineensis, Arecaceae): crecimiento y absorción de nutrimentos
}

\author{
Ángel Madrigal-Valverde' \& Gabriel Garbanzo-León ${ }^{2 *}$ \\ 1. Departamento de Agronomía, Coopeagropal R.L. Potrero Grande, Puntarenas, Costa Rica. \\ 2. Escuela de Agronomía, Centro de Investigaciones Agronómicas. Universidad de Costa Rica. Sabanilla, 11501, San José, Costa Rica; \\ juan.garbanzo@ucr.ac.cr \\ * Correspondencia
}

Recibido 13-X-2017 • Corregido 13-XII-2017 • Aceptado 30-I-2018

\begin{abstract}
Use of industrial waste in prenursery stage of oil palm (Elaeis guineensis, Arecaceae) production: growth and nutrient uptake. Oil palm is an important crop in need of improved production methods. We evaluated industrial waste for variety Deli $x$ Nigeria in pre-nurseries: volumetric mixtures (10 and $20 \%$ ) of oil palm empty fruit bunch (EFB), industrial sludge and industrial ash with soil (80 and $90 \%$ ). Nursery bags were filled with mixture in a restricted randomized block design. The variables were evaluated at 45 and 90 days after planting. We found that $80 \%$ soil $+20 \%$ EFB treatment (S20F) increased leaf length and diameter, biomass, bulb diameter and root length. It also increased nutrient uptake in all mixtures. The concentration of $\mathrm{Mg}, \mathrm{K}$, $\mathrm{P}, \mathrm{Zn}$ and $\mathrm{S}$ was higher than in soil. Industrial waste allows a significant reduction in fertilizer for pre-nurseries.
\end{abstract}

Keywords: Oil palm empty fruit bunch, industrial sludge, industrial ash, oil palm nursery, Deli x Nigeria, compost.
RESUMEN: La palma aceitera es un cultivo importante que necesita mejores métodos de producción. Evaluamos los residuos industriales para la variedad Deli x Nigeria en pre-viveros: mezclas volumétricas (10 y $20 \%$ ) de racimos de frutas vacías de palma aceitera (EFB, por sus siglas en inglés), lodos industriales y cenizas industriales con suelo ( 80 y $90 \%$ ). Las bolsas de vivero se llenaron con mezcla en un diseño de bloques al azar restringido. Las variables se evaluaron a los 45 y 90 días después de la siembra. Encontramos que el tratamiento con $80 \%$ de suelo $+20 \%$ de EFB (S20F) aumentó la longitud y el diámetro de la hoja, la biomasa, el diámetro del bulbo y la longitud de la raíz. También aumentó la absorción de nutrientes en todas las mezclas. La concentración de $\mathrm{Mg}, \mathrm{K}$, $\mathrm{P}, \mathrm{Zn}$ y $\mathrm{S}$ fue mayor que en el suelo. Los desechos industriales permiten una reducción significativa en los fertilizantes para los previveros.

Palabras claves: RFF, lodos industriales, ceniza industrial, viveros palma, Deli x Nigeria, mezclas compost.
Elaeis guineensis Jacq. es un cultivo perenne con un periodo de vida útil entre 25 - 30 años. Esta planta al tener un ciclo prolongado genera producción de racimos frescos después de los 2,5 años hasta su senescencia. Esto lo vuelve muy atractivo para los agricultores además de tener pocos problemas fitosanitarios en comparación a otros cultivos anuales (Chinchilla, 2003; Escobar, 2012). Es por esta razón que se busca establecer en sitios de siembra definitivos las mejores plantas, con el fin de lograr cosechas tempranas y evitar problemas de aclimatación en el sitio (ASD, 2010). Existen aspectos esenciales para garantizar plantas ideales, entre éstas: semilla certificada, plantas sin problemas de patógenos foliares, plantas con excelente nutrición y con buena arquitectura vegetal.

Para garantizar estos aspectos en una palma es necesario realizar manejos de previvero y vivero. En la primera etapa se busca desarrollar las plántulas bajo condiciones controladas y con los mayores cuidados posibles, debido a que las semillas provienen de variedades certificadas y especificadas según las condiciones de la zona, esto las torna de alto valor económico y su precio varía según la variedad. Una vez que las plántulas logran establecer sus primeras hojas lanceoladas pasan a un sistema abierto, donde se desarrollan durante un tiempo aproximado de 12 a 15 meses, este es necesario para obtener un buen desarrollo y resistencia para que puedan tolerar la siembra en sitio definitivos (ASD, 2010). Estos viveros de dos etapas tienen excelentes ventajas, en donde permiten optimar el uso de los recursos, un mejor cuidado a las plantas durante la etapa inicial y garantizar su calidad (Chee, Chiu, Chan, 1998; Negreros-Castillo, ApodacaMartínez \& Mize, 2010). 
La siembra de las plántulas en previveros y viveros se realizan principalmente con suelo. Por su fácil disponibilidad este se lleva a los sitios de viveros y se depositan en sitios específicos para el llenado de bolsas plásticas, permitiendo su fácil manipulación y el desarrollo de palmas (Ortiz \& Fernández, 2000). El suelo usado en los viveros muchas veces no cumple con las características fisicoquímicas ideales para el desarrollo de las plántulas y las raíces (Albertazzi, Chinchilla \& Ramírez, 2009), lo cual incentiva a la búsqueda de enmiendas para aumentar los contenidos nutricionales y brindar características físicas deseables al suelo de las bolsas (ASD, 2010; Fairhurst \& Härdter, 2012).

Trabajos realizados en Costa Rica por Umaña, Chinchilla y Richardson (1991) probaron cuatro tipos de sustratos en viveros de palma de aceite. Estos consistieron en suelo en mezcla (1:3) con granza de arroz, mesocarpio y endocarpio quebrado de frutos de palma de aceite. Los autores encontraron que las mezclas de suelo con mesocarpio fue el más similar al testigo, lo cual recomendaron evaluar nuevas mezclas con distintas materias primas. Con el objetivo de buscar materiales que tenga una baja densidad para facilitar manejo de las bolsas y su crecimiento radicular. Más tarde Rochels (2010) trabajó con distintas dosis de compost en mezcla con suelos en vivero de palmas, donde encontró respuesta positiva en las plántulas al mezclar compost a partir de $2,5 \%$. Así mismo Posso (2010), encontró buenos resultados en plántulas de palma de aceite al aplicar $5 \%$ compost en los suelos de las bolsas, aumentando significativamente la capacidad de intercambio catiónico, materia orgánica y fósforo. Por su parte Garbanzo (2017), evaluó el efecto de dosis crecientes de compost de fibra de racimos vacíos en viveros, donde encontró que las mezclas a 12 y $25 \%$ de compost con el suelo presentaron un desarrollo adecuado de las plántulas por mantener un balance adecuado de nutrimentos en suelos aluviales. Así mismo, el autor mencionó que porcentajes por arriba de $25 \%$ no muestran aumentos en el crecimiento, mejoras en la absorción de nutrimentos y en la tolerancia a enfermedades foliares en viveros de palma aceitera.

En relación con los previveros las investigaciones son escasas debido a que el tiempo de manejo dura aproximadamente tres meses y en ocasiones se usan sustratos empacados (Jiffy) para su desarrollo. Sin embargo, algunos estudios realizados en Malasia con dosis crecientes de compost de mesocarpio de frutas de palma aceitera en un Ultisol con pH de 4,6, demostraron que dosis de $70 \%$ de compost en mezcla con el suelo aumentaron el crecimiento y la absorción de nutrimentos en las plántulas de previveros (Rosenani, Rovica, Cheah \& Lim, 2016). Otros estudios realizados al sur de Benín en África con distintos volúmenes de bolsas, sustratos y fertilización (Akpo, Stomph, Kossou, Omore \& Sruik, 2013), concluyeron que las plántulas no requirieron la adición de fertilizante con la aplicación de estiércol en mezcla con el suelo, debido a que aplicaciones de fertilizante químico potencializarían problemas de salinidad ocasionando muertes de forma indeseable. Estos autores también determinaron que el tamaño de la bolsa no mostró efectos con la adición de fertilizante o mezclas de sustratos en la producción de plántulas.

El objetivo de este trabajo fue evaluar el efecto de dosis de ceniza industrial, lodos industrial y compost de fibra de racimos vacíos en mezclas con suelo sobre el crecimiento y la absorción de nutrimentos en palma aceitera variedad Deli x Nigeria en previveros.

\section{MATERIALES Y MÉTODOS}

El trabajo se desarrolló en los viveros del departamento de Agronomía de la Cooperativa Agroindustrial de Palma de Aceite (Coopeagropal R. L.) en Potrero Grande del cantón de Buenos Aires en Puntarenas. Esta zona se encuentra en la parte alta de la zona pacífico sur de Costa Rica $\left(9^{\circ} 0^{\prime} 3,10^{\prime \prime} \mathrm{N}-83^{\circ} 11^{\prime} 3,37^{\prime \prime} \mathrm{W}\right)$, presenta una elevación aproximada de $110-140 \mathrm{msnm}$, temperatura máxima $31,1^{\circ} \mathrm{C}$ y una mínima de $20,5^{\circ} \mathrm{C}$, humedad relativa de $81 \%$. Durante los registros acumulados presenta una frecuencia de 203 días con lluvias y una precipitación anual de $3416 \mathrm{~mm}$ (IMN, 2015).

Se procedió a buscar un área que fuera plana y con disponibilidad de agua para riego en las instalaciones de los viveros. Se trasladó el suelo y los residuos industriales al área del previvero, donde cada material fue depositado sin ser mezclado y se cubrió con un plástico transparente durante dos semanas. Los residuos industriales consistieron en la recolección de "fibra" de los racimos vacíos, con un año de descomposición en patios al aire libre; la ceniza es un residuo industrial que sale de las calderas producto de los mismos racimos vacíos, se combustiona y el material restante sale seco y listo para el uso; los efluentes llamado en este artículo "lodo industrial", este residuo se extrajo de las lagunas de oxidación, se secaron en pilas durante un año para luego almacenarlos en patios de descomposición. A los tres materiales industriales y al suelo utilizado en esta investigación, se procedió a realizar un análisis químico para determinar la concentración de nutrimentos en cada material (Cuadro 1).

Se procedió a realizar seis mezclas crecientes partiendo de dos bases de suelo ( $80 \%$ y $90 \%$ ) y completando el resto del volumen con los tres residuos industriales $(20 \%$ y $10 \%)$. Para realizar las mezclas con una base de $80 \%$ 
CUADRO 1

Concentración de nutrimentos en suelo y residuos industriales utilizados para las mezclas en previveros de palma aceitera, Coopeagropal 2017.

\begin{tabular}{lccccccccccccc}
\multicolumn{1}{c}{ Mehlich 3 } & $\mathrm{pH} \mathrm{H}_{2} \mathrm{O}$ & \multicolumn{1}{c}{$\mathrm{Cmol}^{(+)} \mathrm{L}^{-1}$} & \multicolumn{1}{c}{$\mathrm{mg} \mathrm{L}^{-1}$} \\
NC & 5,5 & 0,5 & 4 & 1,1 & 0,2 & 5 & 20 & 15 & 1,7 & 0,4 & 10 & 4 & 31 \\
Suelo & 5,4 & 0,4 & 10,5 & 1,9 & 0,1 & 12,9 & 3 & 33 & 1,3 & 10 & 462 & 15 & 26 \\
Fibra & 5,9 & 0,1 & 19,3 & 7,1 & 1,7 & 28,2 & 0,5 & 77 & 11,1 & 14 & 280 & 7 & - \\
Ceniza & 9,9 & - & 1,9 & 1,4 & 4,5 & 7,8 & - & 1570 & 45,3 & 13 & 213 & 22 & - \\
Lodo & 7,3 & 0,1 & 8,9 & 1,5 & 1,0 & 11,5 & 0,9 & 34 & 5,6 & 3 & 53 & 6 & - \\
\hline
\end{tabular}

*Solución extractora Mehlich 3, Laboratorio Suelos y Foliares, Centro de Investigaciones Agronómicas, Universidad de Costa Rica.; NC = nivel crítico para Inceptisoles según Cabalceta \& Molina (2006). CICE = Capacidad de intercambio de Cationes Efectiva (Acidez + Ca + Mg + K.). S.A. = Saturación de Acidez (100* Acidez / CICE).

de suelo, se efectuó mediante una mezcla volumétrica usando un balde de $20 \mathrm{~L}$, aplicando ocho baldes de suelo más dos del material industrial (fibra, lodo o ceniza). Para la mezcla base de $90 \%$ de suelo se procedió a aplicar nueve baldes más uno de los materiales industriales para cada mezcla. Se mezcló homogéneamente y se procedió a colocarlos en bolsas plásticas de $25 \times 20 \mathrm{~cm}$, además para comparar el efecto de los materiales en el previvero se dejó un testigo absoluto con 100\% suelo (Cuadro 2).

CUADRO 2

Mezclas confeccionadas para los tratamientos en las evaluaciones de crecimiento y absorción en previveros de palma aceitera.

\begin{tabular}{crl} 
Tratamiento & Asignatura & \multicolumn{1}{c}{ Mezclas } \\
T1 & S10F & $90 \%$ suelo $+10 \%$ fibra \\
T2 & S10C & $90 \%$ suelo $+10 \%$ ceniza \\
T3 & S10L & $90 \%$ suelo $+10 \%$ lodo \\
T4 & S20F & $80 \%$ suelo $+20 \%$ fibra \\
T5 & S20C & $80 \%$ suelo $+20 \%$ ceniza \\
T6 & S20L & $80 \%$ suelo $+20 \%$ lodo \\
T7 & Suelo & $100 \%$ suelo \\
\hline
\end{tabular}

Una vez llenas se colocaron bloques de $2 \mathrm{~m}^{2}$ ( 84 bolsas por bloque) para un total de 336 unidades experimentales. Así mismo, se sembró una semilla del material Deli $X$ Nigeria en cada bolsa, se presionó fuertemente para su estabilización y garantizar el bienestar de la plúmula, posteriormente se aplicó abundante agua para humedecer el material. El manejo agronómico en el experimento fue similar al de previveros en Coopeagropal R. L., a excepción de la fertilización, el cual no se realizó en el experimento, ya que se buscaba medir el efecto de los productos industriales en la absorción de nutrimentos en las plántulas.

Variables evaluadas: Se realizaron muestreos a los 45 y 90 días después de siembra (dds), para determinar el crecimiento de la planta. Se midió con un vernier (caliper) el diámetro de bulbo que se encuentra a nivel del suelo, en esta sección se limpió el suelo y se midió la parte más ancha del bulbo. Así mismo, con una cinta métrica se midió el largo de raquis que comprendió el largo desde la parte proximal del bulbo hasta el meristemo apical de la hoja. También, se cuantificó el número de hojas totales en las plantas y el área foliar de la hoja, este último fue una aproximación al área de un rombo, debido a que la hoja en estados de previvero es lanceolado similar a un romboide.

Por otro lado, se recolectaron cuatro plantas por tratamiento y se procedió a realizar medidas destructivas a los 45 y 90 dds. Se lavaron con abundante agua y se procedió a separar la parte aérea y raíz. A cada planta se midió el largo de raíz desde la parte basal hasta el meristemo apical del sistema radical, así mismo se pesó cada parte de las plantas para determinar el peso fresco. Luego se secaron durante 48 horas en un horno y posteriormente se pesaron para determinar el peso seco. Ambas partes fueron llevadas al Laboratorio de Suelos y Foliares del Centro de Investigaciones Agronómicas para determinar los contenidos de N, P, N, P, K, Ca, Mg, S, Fe, Zn, Mn, Cu y B.

Para determinar el efecto de los residuos industriales en los contenidos nutricionales del suelo de las bolsas, se realizó un análisis químico a los 90 dds. Se recolectó submuestras en cada bolsa de las repeticiones y se formó una muestra compuesta por cada tratamiento. Estas fueron llevadas al Laboratorio de Suelos y Foliares del 
Centro de Investigaciones Agronómicas para determinar el $\mathrm{pH}$ en agua, acidez intercambiable, $\mathrm{Ca}, \mathrm{Mg}, \mathrm{K}, \mathrm{P}, \mathrm{Fe}, \mathrm{Zn}$, $\mathrm{Mn}, \mathrm{Cu}$, y Al mediante extracciones con Mehlich III según la metodología de Cabalceta y Molina (2006).

Análisis estadístico: El experimento fue constituido por siete tratamientos y cuatro repeticiones con un diseño irrestricto al azar con un acomodo en bloques. Para el análisis de las variables de crecimiento se realizó por medio de una prueba de Shapiro Wilk para verificar la normalidad de los datos, posteriormente se procedió a realizar una prueba de Kruskal Wallis $(a=0,05)$ para los datos no paramétrico y un ANOVA para los datos paramétricos aplicando en esta última la prueba de diferencia mínima significativa (dms) para comparar el efecto de los tratamientos $(a=0,05)$. Los datos fueron calculados mediante el programa estadístico INFOSTAT versión 2016.

\section{RESULTADOS}

Se encontró un aumento significativo $(\alpha=0,05)$ en el crecimiento de las hojas en las plántulas de palma aceitera usando un $20 \%$ de residuos industriales a los 90 dds (Cuadro 3). En el número de hojas a los 45 y 90 dds no se encontró diferencias estadísticas en comparación de todos los tratamientos. Al analizar el largo de la hoja a los 45 dds, se encontró que los tratamientos S20F y Suelo mostraron el mayor largo ( 7,1 y $7,3 \mathrm{~cm})$ significativamente $(\alpha=0,05)$ con respecto al tratamiento $\mathrm{S} 20 \mathrm{C}(5,5 \mathrm{~cm}), \mathrm{S} 20 \mathrm{~L}$ $(5,8 \mathrm{~cm})$ y $\mathrm{S} 10 \mathrm{C}(6,1 \mathrm{~cm})$ respectivamente. Así mismo, a los 90 dds se encontró la misma tendencia donde el tratamiento S20F mostró el mayor largo de hoja $(13,6 \mathrm{~cm})$ en comparación con S10L $(10,8 \mathrm{~cm})$ y S10F $(10,9 \mathrm{~cm})$.

El diámetro de la hoja mostró a los 45 dds el mayor grosor en las plantas sembradas en el Suelo $(2,9 \mathrm{~cm})$ y difirió estadísticamente de $\mathrm{S} 20 \mathrm{~L}(2,3 \mathrm{~cm})$. A los 90 dds se encontró que el tratamiento S20F tuvo el mayor diámetro $(5,0 \mathrm{~cm})$ en comparación a los demás tratamientos, siendo el tratamiento $\mathrm{S} 10 \mathrm{~L}$ el que mostró el menor crecimiento $(3,5 \mathrm{~cm})$.

El área foliar determinada no mostró diferencias estadísticamente significativas a los 45 dds entre los tratamientos. No obstante, al comparar los tratamientos a los 90 dds se encontró que el tratamiento S20F reveló la mayor área foliar $\left(1396,5 \mathrm{~cm}^{2}\right)$ que estadísticamente difiere de varios tratamientos.

La biomasa en las plantas referente a los 45 dds y 90 dds, mostró que la adicción de material residual (fibra, lodo y ceniza) permitió un aumento en la materia seca (Cuadro 4). Al realizar la comparación entre el peso fresco a los 45 dds, no hubo diferencias estadísticas significativas $(a=0,05)$ entre los tratamientos. Sin embargo, al analizar esta variable a los $90 \mathrm{dds}$, el tratamiento S20F obtuvo el mayor peso fresco $(9,4 \mathrm{~g})$ en comparación al resto de los tratamientos. El comportamiento del peso seco fue proporcional al peso fresco, donde a los 45 dds no existieron diferencias estadísticas entre los tratamientos, sin embargo, a los 90 dds el tratamiento S20F mostró la mayor acumulación de biomasa seca $(2,4 \mathrm{~g})$ en comparación al resto de los tratamientos. La obtención del porcentaje de humedad para los tratamientos S10F, Suelo, S10L y

CUADRO 3

Desarrollo de plántulas de palma aceitera bajo la influencia de residuos industriales en mezclas con suelo evaluados en previveros.

\begin{tabular}{lcccccccc}
\multicolumn{1}{c}{ Tratamientos } & $\begin{array}{c}\text { Número } \\
\text { hojas }\end{array}$ & $\begin{array}{c}\text { Largo hoja } \\
(\mathrm{cm})\end{array}$ & $\begin{array}{c}\text { Diámetro hoja } \\
(\mathrm{cm})\end{array}$ & $\begin{array}{c}\text { Área foliar } \\
\left(\mathrm{cm}^{2}\right)\end{array}$ & $\begin{array}{c}\text { Número } \\
\text { hojas }\end{array}$ & $\begin{array}{c}\text { Largo hoja } \\
(\mathrm{cm})\end{array}$ & $\begin{array}{c}\text { Diámetro hoja } \\
\left(\mathrm{cm}^{2}\right)\end{array}$ & $\begin{array}{c}\text { Área foliar } \\
\left(\mathrm{cm}^{2}\right)\end{array}$ \\
DDS & 2,0 & $6,8 \mathrm{ab}^{*}$ & $2,8 \mathrm{ab}$ & 199,7 & 4,0 & $10,9 \mathrm{~b}$ & $4,0 \mathrm{~b}^{+}$ & $919,3 \mathrm{bc}$ \\
S10F & 2,0 & $6,1 \mathrm{bc}$ & $2,8 \mathrm{ab}$ & 174,8 & 4,0 & $12,3 \mathrm{ab}$ & $4,3 \mathrm{~b}$ & $1096,3 \mathrm{ab}$ \\
S10C & 1,8 & $6,4 \mathrm{ab}$ & $2,5 \mathrm{ab}$ & 150,7 & 3,5 & $10,8 \mathrm{~b}$ & $3,5 \mathrm{c}$ & $721,4 \mathrm{c}$ \\
S10L & 2,0 & $7,1 \mathrm{a}$ & $2,8 \mathrm{ab}$ & 206,2 & 4,0 & $13,6 \mathrm{a}$ & $5,0 \mathrm{a}$ & $1396,5 \mathrm{a}$ \\
S20F & 1,8 & $5,5 \mathrm{c}$ & $2,5 \mathrm{ab}$ & 122,7 & 4,0 & $11,4 \mathrm{ab}$ & $4,1 \mathrm{~b}$ & $982,9 \mathrm{bc}$ \\
S20C & 2,0 & $5,8 \mathrm{bc}$ & $2,3 \mathrm{~b}$ & 148,0 & 4,0 & $11,8 \mathrm{ab}$ & $4,1 \mathrm{~b}$ & $1019,5 \mathrm{bc}$ \\
S20L & 1,5 & $7,3 \mathrm{a}$ & $2,9 \mathrm{a}$ & 164,8 & 3,5 & $11,7 \mathrm{ab}$ & $3,9 \mathrm{bc}$ & $828,4 \mathrm{bc}$ \\
Suelo & 0,292 & $<0,05$ & $<0,05$ & 0,444 & 0,081 & $<0,05$ & $<0,001$ & 0,008 \\
P-Value & - & 1,25 & 0,611 & 86,60 & - & 2,32 & 0,423 & 321,2 \\
DMS & & & & & & &
\end{tabular}

* Letras distintas indican diferencia significativa de acuerdo con prueba LSD Test (p.adj= Bonferroni p<0,05) del modelo lineal (LM).

+ Letras distintas indican diferencia significativa de acuerdo con prueba Kruskal wallis $(\alpha<0,05)$ para variables no paramétricas. 


\section{CUADRO 4}

Biomasa y humedad en plántulas de palma aceitera a los 45 y 90 días después de siembra bajo el efecto de mezclas de residuos industriales en previvero.

\begin{tabular}{|c|c|c|c|c|c|c|}
\hline \multirow{2}{*}{ Tratamiento } & Peso fresco $(\mathrm{g})$ & Peso seco (g) & Humedad (\%) & Peso fresco $(\mathrm{g})$ & Peso seco ( $g$ ) & Humedad (\%) \\
\hline & \multicolumn{3}{|c|}{$45 \mathrm{dds}$} & \multicolumn{3}{|c|}{$90 \mathrm{dds}$} \\
\hline S10F & 1,7 & 0,3 & $81,3 b^{+}$ & $5,5 b c^{*}$ & $1,6 \mathrm{bc}$ & $71,6 a$ \\
\hline S10C & 2,1 & 0,4 & $83,0 \mathrm{ab}$ & $7,0 \mathrm{~b}$ & $1,8 a b$ & $73,9 \mathrm{bc}$ \\
\hline S10L & 1,5 & 0,3 & $78,6 \mathrm{~b}$ & $4,4 c$ & $1,2 c$ & $72,7 \mathrm{abc}$ \\
\hline S2OF & 2,0 & 0,4 & $82,3 \mathrm{ab}$ & $9,4 \mathrm{a}$ & $2,4 \mathrm{a}$ & $74,8 \mathrm{~cd}$ \\
\hline S2OC & 1,7 & 0,3 & 84,8 a & $7,3 \mathrm{ab}$ & $1,7 \mathrm{bc}$ & $76,6 \mathrm{~d}$ \\
\hline S20L & 1,8 & 0,3 & $81,6 \mathrm{~b}$ & $6,5 b c$ & $1,7 \mathrm{bc}$ & $73,6 \mathrm{abc}$ \\
\hline Suelo & 1,6 & 0,3 & $78,8 \mathrm{~b}$ & $5,3 \mathrm{bc}$ & $1,5 \mathrm{bc}$ & $72 \mathrm{ab}$ \\
\hline P-Value & 0,722 & 0,770 & 0,04 & 0,003 & 0,03 & 0,001 \\
\hline DMS & 0,811 & 0,130 & - & 2,240 & 0,620 & 2,16 \\
\hline
\end{tabular}

* Letras distintas indican diferencia significativa de acuerdo con prueba LSD Test (p.adj= bonferroni $p<0,05)$ del modelo lineal (LM).

+ Letras distintas indican diferencia significativa de acuerdo con prueba Kruskal wallis $(a<0,05)$ para variables no paramétricas.

S20L reveló diferencias estadísticas $(a=0,05)$ con respecto los demás tratamientos, siendo menor para el tratamiento S10F $(71,6 \%)$.

El desarrollo del sistema radical no mostró problemas en el crecimiento bajo la influencia de los residuos industriales (Fig. 1). El largo de raíz a los 45 dds no mostró diferencia estadística significativa $(\alpha=0,05)$ en comparación al resto de los tratamientos, este efecto fue similar para la evaluación a los 90 dds. Sin embargo, al analizar las tendencias de crecimiento de las raíces, se encontró que las plantas bajo la influencia de un $10 \%$ de fibra y ceniza (S10F y S10C) mostraron mayores tendencias de crecimiento que los demás tratamientos, siendo mayor para la aplicación de $20 \%$ de fibra (S20F).

El crecimiento del diámetro del bulbo en las plántulas de palma aceitera, se encontró un incremento significativo al agregar compost de fibra en mezcla al suelo en las bolsas (Fig. 2). El diámetro de bulbo en los primeros 45 dds no mostró diferencias significativas en comparación al resto de los tratamientos, sin embargo, al analizar este parámetro de crecimiento a los 90 dds se encontró que el tratamiento S20F mostró el mayor diámetro de bulbo $(10 \mathrm{~mm})$ en comparación a las plantas sembradas en Suelo $(8 \mathrm{~mm})$ significativamente $(\alpha=0,05)$.

Las plántulas de palma aceitera bajo la influencia de residuos industriales mostraron un incremento en la absorción de macronutrimentos (Cuadro 5). En la absorción de $\mathrm{N}$ en las plántulas a los 45 dds, mostró el mayor contenido (7,89 $\mathrm{mg}$ planta $\left.^{-1}\right)$ en el tratamiento S10C cuando se comparó con el resto de los tratamientos; no obstante, este comportamiento cambió a los 90 dds donde se encontró que el tratamiento S20F mostró el

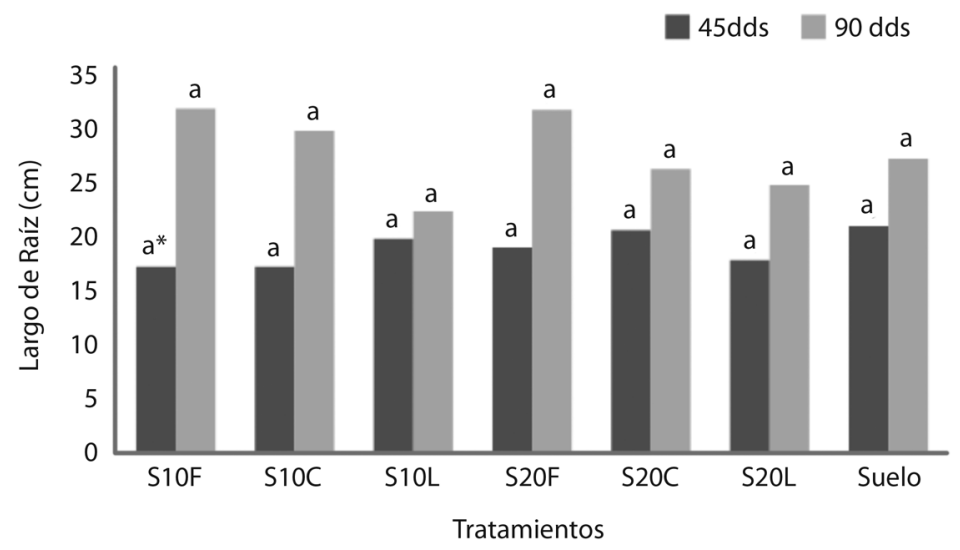

Fig. 1. Desarrollo de la raíz de plántulas de palma aceitera a los 45 y 90 días después de siembra en mezclas de residuos industriales en fase de previveros. * Letras distintas indican diferencia significativa de acuerdo con prueba LSD Test (p.adj= Bonferroni $p<0,05)$. 


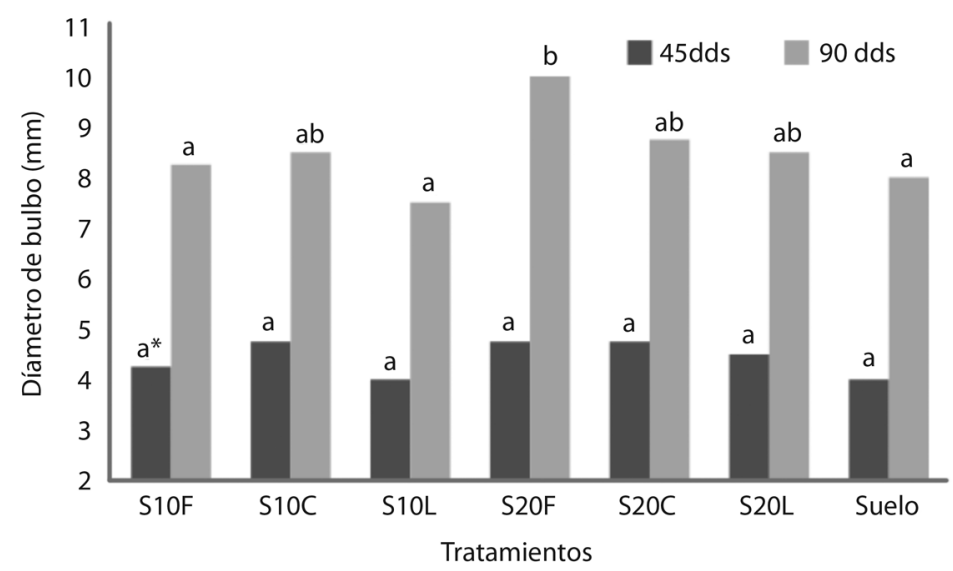

Fig. 2. Diámetro de bulbo en plántulas de palma aceitera a los 45 y 90 días después de siembra, bajo la influencia de residuos industriales en fase de previvero. * Letras distintas indican diferencia significativa de acuerdo con prueba Kruskal wallis $(p<0,05)$ para variables no paramétricas.

mayor contenido $(46,56 \mathrm{mg}$ planta-1) significativamente $(a=0,05)$ en comparación al resto de los tratamientos. La absorción de $\mathrm{P}$ a los 45 dds no tuvo diferencias estadísticas entre los tratamientos, sin embargo, a los 90 dds el tratamiento $\mathrm{S} 10 \mathrm{C}$ presentó la mayor absorción $(5,15 \mathrm{mg}$ planta ${ }^{-1}$ ) en comparación al resto de los tratamientos.

El K manifestó a los 45 dds la mayor acumulación en los tratamientos S2OL (2,23 mg planta- $\left.{ }^{-1}\right)$ y Suelo $(2,19 \mathrm{mg}$ planta $\left.{ }^{-1}\right)$, mientras que el comportamiento a los $90 \mathrm{dds}$ mostró una mayor acumulación en el tratamiento S20F donde tuvo la mayor absorción (14,36 $\left.\mathrm{mg} \mathrm{planta}^{-1}\right)$. El Ca no reveló diferencias entre los tratamientos a los $45 \mathrm{dds}$, pero a los 90 dds el tratamiento S20F presentó la mayor absorción (7,55 mg planta-1) en comparación al resto de los tratamientos. El Mg fue el nutrimento de mayor absorción en las plántulas de previvero, siendo el tratamiento $\mathrm{S} 10 \mathrm{C}$ el de mayor acumulación $\left(7,02 \mathrm{mg}\right.$ planta $\left.{ }^{-1}\right)$ a los 45 dds. A los 90 dds, el tratamiento S20F mostró la mayor acumulación (49,46 mg planta-1 ${ }^{-1}$ en comparación al resto de los tratamientos. Por último, la absorción de $S$ no reveló diferencias estadísticas significativas a los 45 dds, cuando se compararon los tratamientos. A los 90 dds se encontró que el tratamiento S20F presentó la mayor acumulación de $S(4,09 \mathrm{mg}$ planta-1) en relación con los demás tratamientos.

Las plantas de palma aceitera en previveros incrementaron la absorción de micronutrimentos al mezclar residuos industriales con el suelo de las bolsas (Cuadro 5). La absorción de $\mathrm{Zn}$ a los 45 dds no mostró diferencias entre los tratamientos; sin embargo, a los 90 dds se encontró la mayor absorción significativamente $(\alpha=0,05)$ en el tratamiento S2OF $\left(0,078\right.$ mg planta $\left.^{-1}\right)$. El B no presentó una absorción marcada entre los tratamientos a los 45 dds, pero al analizar la acumulación a los 90 dds, se encontró que los tratamientos S20F, S20C y S10C tuvieron las mayores absorciones en comparación al resto de los tratamientos.

La absorción de Fe, fue significativa a partir de los 45 dds, donde el tratamiento $\mathrm{S} 20 \mathrm{C}$ se destaca con relación a los demás tratamientos, así mismo a los 90 dds, se encontró la mayor acumulación de este micronutrimento en el tratamiento S2OF (2,193 $\mathrm{mg}$ planta $\left.{ }^{-1}\right)$. El Cu con el tratamiento $\mathrm{S} 10 \mathrm{~L}$ se obtuvo la mayor acumulación a los $45 \mathrm{dds}$, mientras que a los $90 \mathrm{dds}$, el tratamiento S20F $(0,051 \mathrm{mg}$ planta-1) reveló la mayor acumulación. El Mn no presentó diferencias estadísticas a los 45 dds, pero a los 90 dds, el tratamiento S20F mostró la mayor absorción (0,022 mg planta $^{-1}$ ) en comparación a los otros tratamientos.

La concentración residual de los nutrimentos aportados por las mezclas de residuos industriales se incrementó en el tiempo con las distintas dosis (Cuadro 6). El pH en las mezclas aumentó en comparación con el Suelo, el cual estuvo por debajo del nivel crítico. Así mismo, el tratamiento Suelo mostró problemas de acidez intercambiable en comparación a las mezclas. En cuanto a los contenidos de $\mathrm{Mg}$ y el K, éstos se incrementaron en comparación al tratamiento Suelo, principalmente para las mezclas con presencia de ceniza (S10C y S20C) y en un estado intermedio para las mezclas con presencia de fibra (S10F y S20F). El mismo efecto se encontró para los contenidos de $\mathrm{P}$ y $\mathrm{Zn}$, donde las mezclas con presencia de ceniza (S10C y S20C) incrementaron los contenidos de estos elementos. Por último, al comparar la concentración de nutrimentos al inicio del experimento (Cuadro 1) con los contenidos a los 90 dds, se determinó que el suelo usado mostró deficiencias de K, Zn y no presentaba problemas de acidez intercambiable, sin embargo, al ser 
CUADRO 5

Absorción de nutrimentos en plantas de palma aceitera sembradas en mezclas de residuos industriales en fase de previvero.

\begin{tabular}{|c|c|c|c|c|c|c|c|c|c|c|c|}
\hline \multirow{3}{*}{ Tratamientos } & $\mathrm{N}$ & $P$ & K & $\mathrm{Ca}$ & $\mathrm{Mg}$ & $S$ & $\mathrm{Zn}$ & B & $\mathrm{Fe}$ & $\mathrm{Mn}$ & $\mathrm{Cu}$ \\
\hline & \multicolumn{11}{|c|}{$45 \mathrm{dds}$} \\
\hline & \multicolumn{11}{|c|}{ mg planta $^{-1}$} \\
\hline S10F & $6,65 a^{+}$ & 0,54 & $1,77 a b$ & 0,85 & $5,43 a b$ & 0,60 & 0,007 & $0,003 b^{*}$ & $0,327 b^{+}$ & 0,016 & $0,007 a^{*}$ \\
\hline S10C & $7,89 a$ & 0,68 & $2,03 a b$ & 1,02 & $7,02 \mathrm{a}$ & 0,61 & 0,008 & $0,005 \mathrm{a}$ & $0,325 b$ & 0,016 & $0,008 a b$ \\
\hline S10L & $5,21 b$ & 0,51 & $2,17 a$ & 1,09 & 4,12 bc & 0,63 & 0,009 & $0,003 \mathrm{a}$ & $0,360 \mathrm{~b}$ & 0,019 & $0,017 \mathrm{~b}$ \\
\hline S2OF & $6,72 a b$ & 0,71 & $2,08 a b$ & 1,23 & $5,95 a b$ & 0,72 & 0,011 & $0,004 \mathrm{a}$ & $0,414 b$ & 0,025 & $0,008 a b$ \\
\hline$S 20 C$ & $5,69 a b$ & 0,54 & $1,29 \mathrm{~b}$ & 0,83 & $5,90 \mathrm{ab}$ & 0,47 & 0,007 & $0,004 a b$ & $0,624 a$ & 0,013 & $0,007 \mathrm{a}$ \\
\hline S20L & $5,55 a b$ & 0,57 & $2,23 \mathrm{a}$ & 1,09 & 4,77 bc & 0,67 & 0,009 & $0,003 a$ & $0,401 \mathrm{~b}$ & 0,016 & $0,009 a b$ \\
\hline Suelo & $6,16 a b$ & 0,51 & $2,19 a$ & 0,96 & $2,93 \mathrm{C}$ & 0,61 & 0,008 & $0,003 \mathrm{a}$ & $0,376 b$ & 0,022 & $0,009 a b$ \\
\hline P-Value & $<0,05$ & 0,36 & $<0,05$ & 0,40 & $<0,01$ & 0,59 & 0,09 & $<0,01$ & 0,02 & 0,31 & $<0,05$ \\
\hline \multirow[t]{3}{*}{ DMS } & 2,63 & 0,22 & 0,85 & 0,40 & 1,93 & 0,25 & 0,007 & - & 0,17 & - & - \\
\hline & \multicolumn{11}{|c|}{$90 \mathrm{dds}$} \\
\hline & \multicolumn{11}{|c|}{ mg planta-1 } \\
\hline $\mathrm{S} 10 \mathrm{~F}$ & $26,00 \mathrm{c}$ & $3,02 \mathrm{~cd}$ & 10,24 b & $5,19 \mathrm{bcd}$ & $27,80 \mathrm{bc}$ & $2,99 a b c$ & $0,041 \mathrm{~cd}$ & $0,015 a b^{*}$ & 1,738ab & $0,104 b c^{*}$ & $0,036 b c^{*}$ \\
\hline $\mathrm{S} 10 \mathrm{C}$ & $33,39 b c$ & $5,15 a$ & $10,26 \mathrm{~b}$ & $6,80 a b$ & 37,14 b & $3,31 \mathrm{abc}$ & $0,059 a b$ & $0,017 b$ & $1,695 \mathrm{ab}$ & $0,048 \mathrm{a}$ & $0,035 b c$ \\
\hline S10L & $30,06 \mathrm{c}$ & $1,91 \mathrm{~d}$ & $7,65 \mathrm{~b}$ & $3,43 d$ & $19,56 \mathrm{c}$ & $2,24 \mathrm{c}$ & $0,028 d$ & $0,010 \mathrm{a}$ & $1,298 \mathrm{~b}$ & $0,048 \mathrm{a}$ & $0,016 a$ \\
\hline $\mathrm{S} 2 \mathrm{~F}$ & $46,56 \mathrm{a}$ & $4,76 \mathrm{ab}$ & $14,36 \mathrm{a}$ & $7,55 \mathrm{a}$ & $49,46 \mathrm{a}$ & $4,09 a$ & $0,078 \mathrm{a}$ & $0,022 \mathrm{~b}$ & $2,193 \mathrm{a}$ & $0,124 \mathrm{bc}$ & $0,051 \mathrm{c}$ \\
\hline S20C & $30,57 \mathrm{c}$ & $3,93 a b c$ & $8,00 \mathrm{~b}$ & $6,15 a b c$ & $37,39 \mathrm{~b}$ & $2,85 \mathrm{bc}$ & $0,059 \mathrm{bc}$ & $0,018 \mathrm{~b}$ & $1,517 \mathrm{ab}$ & $0,041 \mathrm{a}$ & $0,043 b c$ \\
\hline S20L & $43,62 a b$ & 3,79 bc & $11,13 a b$ & $4,69 \mathrm{~cd}$ & $33,11 \mathrm{~b}$ & $3,83 a b$ & 0,046 bc & $0,016 a b$ & $1,295 \mathrm{~b}$ & $0,060 \mathrm{ab}$ & $0,021 \mathrm{ab}$ \\
\hline Suelo & $23,17 \mathrm{c}$ & $2,93 \mathrm{~cd}$ & $9,19 \mathrm{~b}$ & $5,77 a b c$ & $18,26 \mathrm{c}$ & $2,61 \mathrm{c}$ & $0,040 \mathrm{~cd}$ & $0,013 a b$ & 1,651 ab & $0,138 c$ & $0,031 \mathrm{abc}$ \\
\hline P-Value & $<0,01$ & $<0,01$ & 0,01 & 0,01 & $<0,01$ & $<0,05$ & $<0,01$ & $<0,05$ & $<0,05$ & $<0,01$ & $<0,01$ \\
\hline DMS & 11,9 & 1,27 & 3,48 & 2,07 & 11,80 & 1,20 & 0,02 & - & 0,71 & - & - \\
\hline
\end{tabular}

+Letras distintas indican diferencia significativa de acuerdo con prueba de $\mathrm{dms}(\alpha=0,05) .{ }^{*}$ Letras distintas indican diferencia significativa de acuerdo con prueba Kruskal wallis $(p<0,05)$ para variables no paramétricas.

CUADRO 6

Concentración química de nutrimentos en mezclas de residuos industriales con suelo a los 90 días después de siembra de plántulas de palma aceitera en fase de previvero.

\begin{tabular}{lccccccccccccc} 
Mehlich 3 & $\mathrm{pH} \mathrm{H}_{2} \mathrm{O}$ & \multicolumn{1}{c}{$\mathrm{Cmol}^{(+)} \mathrm{L}^{-1}$} & \multicolumn{1}{c}{$\mathrm{mg} \mathrm{L}^{-1}$} \\
NC & & 0,5 & 4 & 1,1 & 0,2 & 5 & 20 & 15 & 1,7 & 0,4 & 10 & 4 & 31 \\
S10F & 5,5 & 0,24 & 10,84 & 2,21 & 0,30 & 13,59 & 2 & 43 & 1,8 & 8 & 473 & 18 & 22 \\
S10C & 6,7 & 0,13 & 11,99 & 4,27 & 2,35 & 18,74 & 0,7 & 245 & 6,8 & 13 & 411 & 13 & 27 \\
S10L & 5,7 & 0,13 & 10,78 & 1,74 & 0,22 & 12,87 & 1 & 34 & 1,8 & 9 & 370 & 13 & 25 \\
S2OF & 5,3 & 0,24 & 10,30 & 2,32 & 0,42 & 13,28 & 2 & 60 & 2,5 & 8 & 428 & 11 & 25 \\
S20C & 7,4 & 0,14 & 12,26 & 5,34 & 4,20 & 21,94 & 0,6 & 404 & 11,8 & 13 & 392 & 17 & 34 \\
S20L & 6,0 & 0,13 & 14,18 & 1,81 & 0,28 & 16,40 & 0,8 & 41 & 3,5 & 11 & 382 & 11 & 33 \\
Suelo & 5,3 & 0,50 & 8,06 & 1,56 & 0,12 & 10,24 & 5 & 26 & 1,2 & 7 & 406 & 10 & 18 \\
\hline
\end{tabular}


mezclados con los distintos materiales industriales, llevaron los contenidos por arriba del nivel crítico en estos nutrimentos y mostró problemas de acidez intercambiable.

Los valores debajo de cada elemento corresponden con los Niveles Críticos generales para la solución extractora usada. $\mathrm{CICE}=$ Capacidad de intercambio de Cationes Efectiva=Acidez+Ca+Mg+K. SA=Porcentaje de Saturación de Acidez $=($ Acidez/CICE $) * 100$.

\section{DISCUSIÓN}

Las plántulas de palma aceitera mostraron una mayor respuesta en el crecimiento a la aplicación de $20 \%$ de fibra de racimos vacíos (S20F) en el suelo. El efecto de la adición de fibra de palma aceitera en esta proporción mostró un efecto significativo en comparación a un 10\% (S10F), lo que probablemente este efecto incremente la disponibilidad de nutrimentos, mejore los balances, aumente la retención de humedad y aumente los microorganismos en las mezclas (Segura, 2001; Odeoluwa \& Adeoye, 2008; Reyes, Rodríguez, Peña, Bastidas \& Ibarra, 2009; Garbanzo, Molina, Ramírez \& Serrano, 2016). Efectos similares encontró Rosenani et al. (2016) donde evaluaron dosis crecientes de compost de fibra y determinaron que a un $70 \%$ de fibra en mezcla con suelo (ultisol) incrementó el crecimiento de las plantas en previvero. Así mismo, Garbanzo (2017) demostró que aplicaciones por arriba de $25 \%$ con suelo (inceptisol) en viveros de palma aceitera, no presentaron efectos significativos en el crecimiento y la tolerancia a enfermedades en palma aceitera. Esto concuerda con lo encontrado en este trabajo con relación con las aplicaciones de fibra compostada en inceptisoles.

El mayor efecto del crecimiento en las plantas se obtuvo significativamente a los 90 dds. Es probable que las plántulas a los 45 dds utilizaron las reservas de las semillas y no directamente del aporte que brindaba nutricionalmente el suelo. El efecto de crecimiento en las plántulas usando energía de la semilla después de la germinación, está bien demostrado en la fisiología de las semillas (Flores, 1989; Herrera, Alizaga, Guevara \& Jimenez, 2006). Así mismo, los efectos de las mezclas aumentaron el crecimiento del sistema radical, mostrando tendencias no significativas de mayor crecimiento bajo la influencia específica de cada residuo industrial. Rochels (2010) y Coto, Chinchilla, Bulgarelli y Palma (2002) indicaron que el número y tamaño de las raíces es un indicador del crecimiento y la salud de las mismas, permitiendo aumentar la absorción de agua y nutrimentos en las plántulas. Este efecto también fue estudiado por Albertazzi et al. (2009) y Galindo y Romero (2012) en palma aceitera, quienes mencionaron que la dinámica de las raíces en el tiempo y la distribución depende del sitio, especifícame del efecto integral con relación a la fertilidad, parámetros físicos y biológicos del suelo para la siembra de palma aceitera.

Las variables de crecimiento determinan la calidad de las plántulas antes del trasplante en los viveros. El diámetro de bulbo determina directamente el vigor de las plántulas, es decir, entre mayor sea el crecimiento del diámetro del bulbo mayor será el vigor. Además, en comparación al peso seco de la plántula, esta demostró un efecto proporcional al diámetro del bulbo, siendo el tratamiento S20F el que presentó el mayor incremento entre ambas variables en comparación al resto de las mezclas evaluadas. Investigaciones realizadas en viveros han relacionado el peso de la planta y el diámetro de bulbo en cuantificar problemas de calidad en estos biosistemas. Un caso particular e importante en viveros son las enfermedades lo cual estas reducen el vigor de la planta cuando se comparan plantas sanas de las enfermas (Ulloa \& Ramírez, 2011; Ulloa \& Serrano 2012). Este experimento no mostró problemas de enfermedades, lo que se puede determinar que el vigor proviene directamente del efecto de los residuos industriales usados en el suelo. A esto se suma la importancia de evaluar otras variables de crecimiento, como fueron el largo, ancho de la hoja y el área foliar; estas variables se encuentran asociadas directamente con la mayoría de los procesos fisiológicos, que forman parte del análisis de crecimiento, fotosíntesis, transpiración, interceptación de luz y el balance de energía (Cabezas, Peña, Duarte, Colorado \& Lora, 2009; ASD, 2010).

La acumulación de nutrimentos se incrementó significativamente para el tratamiento con $20 \%$ de fibra en mezcla con el suelo (S20F). Este efecto es proporcional con el peso seco y la concentración de nutrimentos evaluados en la parte aérea y raíz de las plántulas, demostrando una relación proporcional en ambas variables. Esto concuerda con Coto et al. (2002) quienes mencionaron que la acumulación de peso seco refleja la capacidad de crecimiento que obtiene una planta, ya que mantiene una actividad fotosintética alta y por ende una absorción adecuada. Sin embargo, en algunos casos específicos que en esta investigación se encontró, fue que algunas mezclas proporcionaron una mayor acumulación de $\mathrm{P}$ a los 90 dds, como es el caso de la mezcla S10C, pero no fue proporcional al doble de su aplicación (S20C). Quizás este efecto sea por la concentración de P que mostraron en la parte aérea, debido a que no hubo diferencias estadísticas en el crecimiento a los 45 dds (Cuadro 4). Por otro lado, el Mn mostró la mayor absorción en comparación al resto de los tratamientos. Es probable que el efecto de absorción a los 90 dds sea producto de los contenidos 
de nutrimentos que aportan los residuos industriales (Cuadro 1). Según Torres, Acosta y Chinchilla. (2004) indicaron que al adicionar residuos de materiales orgánicos se pueden llegar a obtener aportes significativos en los contenidos de N, P, K, Ca, Mg y micronutrimentos en el suelo, lo que concuerda también con los estudios de Odeoluwa y Adeoye (2008). Sin embargo, se ha encontrado que la aplicación en exceso de materiales orgánicos en viveros de palma aceitera no necesariamente incremente la absorción de nutrimentos, debido a que depende principalmente del balance y la solubilidad de los mismos que se logre obtener con el porcentaje adecuado de los materiales orgánicos al suelo (Galindo \& Romero, 2012; Garbanzo et al., 2016).

Al analizar el efecto de la absorción de nutrimentos en las plantas es muy probable que este efecto sea producto del aporte de los nutrimentos que dieron las mezclas al suelo. Los valores de $\mathrm{pH}$ en los residuos industriales (Cuadro 6) presentaron altos contenidos en la ceniza $(9,9)$ y lodo (7.3), aumentando los valores en el suelo en el tiempo. Este efecto probablemente aumentó los problemas con la disponibilidad de los nutrimentos, principalmente para las mezclas con ceniza donde el $\mathrm{pH}$ incremento de 5,4 a 6,7. Está bien demostrado que al aumentar el pH en el suelo muchos de los microelementos pierden su solubilidad y son más difíciles de ser absorbidos por las plantas (Posso, 2010), este efecto también se ha encontrado en viveros (Garbanzo et al., 2016). Es probable que la mezcla de $20 \%$ de fibra brindara un $\mathrm{pH}$ adecuado para que muchos de los nutrimentos estuvieran disponibles para las plantas, haciendo un balance en la absorción de estos. Las cenizas y los lodos industriales son muy ricos en nutrimentos, aportando contenidos significativos al suelo. Sin embargo, la cantidad de nutrimentos que requieren las plántulas en previveros son pequeñas en comparación a los viveros y palma adulta (Umaña, Chinchilla \& Richardson, 1990; Ramírez \& Muñoz, 2010; Ibarra \& Reyes, 2015), demostrando que una adecuada aplicación de materiales industriales puede incrementar el crecimiento de las plántulas en comparación al uso de solo suelo y que para esta investigación sustituyó completamente el uso de fertilizantes.

Se concluye que la mezcla de $20 \%$ de compost de fibra de racimos vacíos de fruta con el suelo mejoraron las características químicas, aumentó el crecimiento y la absorción de nutrimentos en previveros de palma aceitera. Así mismo, se encontró que los residuos industriales (lodo y ceniza) son materiales muy ricos en nutrimentos, principalmente en $\mathrm{P}$. Estos residuos podrían ser utilizados para futuras investigaciones en fase de viveros debido a que su influencia en las características químicas del suelo podría mejorar la absorción de los nutrimentos por las plantas, lo que probablemente reduciría la aplicación de fertilizantes en fase de viveros.

\section{AGRADECIMIENTOS}

Agradecemos al Departamento de Agronomía de Coopeagropal R. L. por el financiamiento de los análisis químicos de suelos y tejidos, que se realizaron en esta investigación; al Centro de Investigaciones Agronómicas (CIA), la Escuela de Agronomía de la Universidad de Costa Rica (UCR) y al departamento de semillas de ASD de la Compañía Palmatica S. A. por brindar la variedad que se evaluó en esta investigación

\section{REFERENCIAS}

Akpo, E., Stomph, T., Kossou, D., Omore, A., \& Sruik, P. (2013). Effects of nursery management practices on morphological quality attributes of tree seddlings at planting: the case of oil palm (Elaeis guineensis Jacq.). Forrest Ecology and Management, 324(2014), 28-36. doi: 10.1016/j. foreco.2014.03.045

Albertazzi, H., Chinchilla, C., \& Ramírez, C. (2009). Características del suelo y desarrollo radical en palma aceitera joven (Elaeis guineensis jacq) en sitios afectados por pudriciones del cogollo. ASD Oil Palm Papers, 33(1), 17-32.

ASD. (2010). Establishment and management of oil palm nurseries (en línea). Coto 47, Costa Rica. Recuperado de http:// www.asd-cr.com/images/nurserie-guide.pdf

Cabalceta, G., \& Molina, E. (2006). Niveles críticos de nutrimentos en suelos de Costa Rica utilizando la solución extractora Mehlich 3. Agronomía Costarricense, 30(2), 31-44.

Cabezas, M., Peña, F., Duarte, H., Colorado, J., \& Lora, S. (2009). Un modelo para la estimacion del area foliar en tres especies forestales de forma no destructiva. Revista actualidad y divulgación Científica, 12(1), 121-130.

Chee, K., Chiu, S., \& Chan, S. (1998). Plántulas de pre-vivero cultivadas en bandejas de macetas. PALMAS, 19(4), 51-54.

Chinchilla, C. (2003). Manejo integrado de problemas fitosanitarios en palma aceitera Elaeis guineensis en América Central. Manejo integrado de plagas y agroecologia, 67, 69-82.

Coto, E., Chinchilla, C., Bulgarelli, J., \& Palma, T. (2002). Crecimiento vegetativo en previvero de cinco cruces comerciales de palma aceitera (Elaeis guineensis jacq). ASD Oil Palm Papers, 23(1), 14-19.

Escobar, R. (2012). ¿Por qué cultivar palma aceitera?. ASD Oil Palm Papers, 39(1), 13-26.

Fairhurst, T., \& Härdter, R. (2012). Palma aceitera. Manejo para rendimientos altos y sostenibles. Ecuador: International 
Plan Nutrition Institute (IPNI) y International Potash Institute (IPI).

Flores, E. (1989). La planta: estructura y función. Cartago, Costa Rica: Tecnológica de Costa Rica.

Galindo, T., \& Romero, H. (2012). Compostaje de subproductos de la agroindustria de palma de aceite en Colombia: estado del arte y perspectivas de investigación. Cenipalma, Bogotá, Colombia: Molher Impresores.

Garbanzo, G. (2017). Evaluación de distintas fuentes nutricionales en la tolerancia de enfermedades foliares en viveros de palma aceitera (Elaeis guineensis Jacq) en Puntarenas, Costa Rica (Tesis de Maestría). Universidad de Costa Rica, San José, Costa Rica.

Garbanzo, G., Molina, E., Ramírez, F., \& Serrano, E. (2016). Aumento de la tolerancia a enfermedades en viveros de palma aceitera mediante fuentes de Silicio. Memoria del XXI Congreso Latinoamericano de la Ciencia de Suelo, Quito, Ecuador.

Herrera J., Alizaga, R., Guevara, E., \& Jimenez, V. (2006). Germinación y crecimiento de la planta. Fisiología de la producción de los cultivos tropicales. San José, Costa Rica: Universidad de Costa Rica.

Ibarra-Ruales, L., \& Reyes-Cuesta, R. (2015). Crecimiento en vivero de las palmas aceiteras Elaeis oleífera $x$ Elaeis guineensis y Elaeis guineensis x Elaeis guineensis en Tumaco Colombia. Corpoica Ciencia y Tecnología Agropecuaria, 16(2), 239-250.

IMN(Instituto meteorológico Nacional de Costa Rica). (2015). Registro de estación meteorológica de Buenos Aires, Puntarenas durante el periodo de 185 - 2016 (en línea). Recuperado de https://www.imn.ac.cr/web/imn/inicio

Negreros-Castillo, P., Apodaca-Martínez, M., Mize, C. (2010). Efecto de sustrato y densidad en la calidad de plántulas de cedro, caoba y roble. Madera bosques, 16(2),7-18.

Odeoluwa, O., \& Adeoye, O. (2008). Potential of oil palm empty fruit bunch (EFB) as fertilizer in oil palm (Elaeis guineensis jacq) nurseries. 16th IFOAM organic world congress (en línea), Italia. Recuperado de http://orgprints.org/12808/

Ortiz, R., \& Fernández, O. (2000). Cultivo de palma aceitera. San José, Costa Rica: EUNED.

Posso, J. (2010). Evaluación de diferentes dosis de compost y lombricompuesto aplicado al suelo de vivero de palma aceitera (Elaeis guinensis) (Tesis de maestría). Universidad Nacional de Colombia. Bogotá, Colombia.
Ramírez, F., \& Muñoz, F. (2010). Curva de nutrientes para la etapa de vivero de tres materiales de palma aceitera (Elaeis guineensis Jacq.). Congreso Ecuatoriano de la Ciencia del Suelo, Santo Domingo, Ecuador.

Reyes, R., Rodríguez, N., Peña, E., Bastidas, S., \& Ibarra, N. (2009). Crecimiento en vivero de materiales comerciales de palma aceitera (Elaeis guineensis Jacq.) en Tumaco, Colombia. Revista Corpoica-Ciencia y Tecnología, 9(2), 12-18.

Rochels, B. (2010). Respuesta de la palma de aceite (Elaeis guinensis) a la aplicación de biofertilizantes en previvero (Tesis de grado). Universidad Nacional de Colombia, Valledupar, Colombia.

Rosenani, A. B., Rovica, R., Cheah, R. M., \& Lim, C. T. (2016). Growth performance and nutrient uptake of oil palm seedling in prenursery stage as influenced by Oil Palm waste compost in growing media. International Journal of Agronomy, 2016(1),1 - 8. doi: 10.1155/2016/6930735

Segura J. (2001). Uso de dos bioensayos para estimar el efecto residual y el valor nutricional de un compost hecho de fibra de racimos vacíos de la palma aceitera (Elaeis guineensis Jacq) (Tesis Licenciatura). Universidad de Costa Rica. San José, Costa Rica..

Torres, R., Acosta, A., \& Chinchilla, C. (2004). Proyecto comercial de compostaje de los desechos agroindustriales de la palma aceitera. Palmas, 25(1), 377-387.

Ulloa, M., \& Serrano, E. (2012). Prueba de sensibilidad in vitro de Colletotrichum gloeosporioides y Curvularia lunata, principales agentes causales del Complejo de Necrosis Foliar (CNF) a diferentes fungicidas. Boletín Mensual de la Unidad de Investigación de Palma Tica S.A. y Cukra Development Corporation, Costa Rica.

Ulloa, M., Ramírez, F. (2011). Resultado del diagnóstico sobre la necrosis foliar del vivero Coto 49. Boletín Mensual de la Unidad de Investigación de Palma Tica S.A. y Cukra Development Corporation, Costa Rica.

Umaña C., Chinchilla, C., \& Richardson, D. (1990). Material de desarrollo avanzado en viveros de palma aceitera (Elaeis guineensis) II. Condiciones del sustrato. Turrialba, 40(1), 440-451.

Umaña, C., Chinchilla, C., \& Richardson, D. (1991). Material de desarrollo avanzado en viveros de palma aceitera (Elaeis guinensis Jacq.) II. Condiciones del sustrato. Turrialba, 40(4), 440-451. 